\title{
Synthesis of SnO Nanopatricles-A Hydrothermal Approach
}

\section{Eppakayala Janardhan'1, Mettu Maheshwar Reddy¹, Pendyala Venkat Reddy ${ }^{1}$, Madireddy Jaipal Reddy ${ }^{*}$}

\author{
${ }^{1}$ Sreenidhi Institute of Science and Technology, Hyderabad, India \\ ${ }^{2}$ Palamuru University, Mahaboobnagar, India \\ Email:*janardhanphy@yahoo.com
}

How to cite this paper: Janardhan, E., Reddy, M.M., Reddy, P.V. and Reddy, M.J. (2018) Synthesis of SnO Nanopatricles-A Hydrothermal Approach. World Journal of Nano Science and Engineering, 8, 33-37. https://doi.org/10.4236/winse.2018.82002

Received: June 6, 2018

Accepted: June 26, 2018

Published: June 29, 2018

Copyright @ 2018 by authors and Scientific Research Publishing Inc. This work is licensed under the Creative Commons Attribution International License (CC BY 4.0).

http://creativecommons.org/licenses/by/4.0/ (c) (i) Open Access

\begin{abstract}
SnO nanoparticles were prepared by addition of $\mathrm{HCl}$ to tin oxide solution. The synthesis process is simplified for $\mathrm{SnO}$ nuclei. We herein report a better-defined and simple procedure for synthesis of SnO particles in a simple hydrothermal process.
\end{abstract}

\section{Keywords}

Tin Oxide, XRD, SEM, Nanostructure, Precipitation, Urea

\section{Introduction}

Metal and metal oxide nanoparticles, differing from their bulk analogs in chemical, thermal, optical, magnetic and other properties, are widely used in catalysis, medicine, electronics and other fields. Many different methods of nanoparticle synthesis with the use of supercritical fluids (SCF) have been suggested, in particular, the reverse micelle, rapid expansion and hydrothermal synthesis methods [1] [2] and [3]. Among these methods hydrothermal process has the best advantages and possibilities for synthesis of metal and metal oxide nanoparticles [4] [5]. Metal oxide nanomaterials can completely degrade the contaminants with sunlight or UV radiation at room temperature and do not cause pollution [6]. These oxide nanomaterials include $\mathrm{TiO}_{2}$ [7], $\mathrm{ZnO}$ [8], $\mathrm{SnO}$ [9] [10] and [11], and $\mathrm{Fe}_{2} \mathrm{O}_{3}$ [12].

$\mathrm{SnO}$ is an important semiconductor material with excellent chemical and physical performances. As an effective photocatalyst, $\mathrm{SnO}$ nanostructures can photodegrade organic pollutants to other nontoxic small molecules. In this work, we report on the synthesis of SnO nanoparticles using a one-pot hydro- 
thermal method.

\section{Synthesis of SnO Nanoparticles}

The synthesis of SnO Nano particles was carried out by conventional Hydrothermal protocol, $\mathrm{SnCl}_{2} \cdot 2 \mathrm{H}_{2} \mathrm{O}$ and Dil. $\mathrm{HCl}$ was used as synthesizing material. In a typical Procedure stock solutions of $0.1 \mathrm{M}(2.3 \mathrm{~g}) \mathrm{SnCl}_{2} \cdot 2 \mathrm{H}_{2} \mathrm{O}$, solution was prepared in $50 \mathrm{ml}$ of $1.0 \mathrm{M} \mathrm{HCl}$ under stirring. To this stock solution $250 \mathrm{ml}$ of $\mathrm{SnCl}_{2}(0.1 \mathrm{M})$ solution prepared in appropriate amount of urea was added under continuous stirring in order maintain the $\mathrm{pH}$ of reactants as 9 . The solution was transferred into Teflon lined autoclave and maintained at $150^{\circ} \mathrm{C}$ for $1 \mathrm{hr}$ under autogenous pressure. It was then allowed to cool naturally to room temperature. After the reaction was complete, the resulting white solid product was washed with distilled water to free the precipitates, filtered and then dried in air in a laboratory oven at $60^{\circ} \mathrm{C}$. The same was shown in flow chart Figure 1 .

\section{Characterization Studies}

\subsection{XRD Study of SnO Nanoparticles}

The XRD results reveal the presence of tetragonal stannous Oxide as shown in Figure 2. with orientation in (001), (101), (110), (002), (200), (112), (211), (202) and (103) planes at 18.2, 29.8, 33.2, 37.1, 44.3, 47.8, 50.7, 57.3 and 62.5 theta values corresponding to $\mathrm{SnO}$ and these values well matches with JCPDD No. 36-1451 data.

\subsection{FT-IR Spectrum of SnO Nanopartilces}

The FT-IR spectrum Figure 3 of the SnO nanoparticles, the absorption peaks at

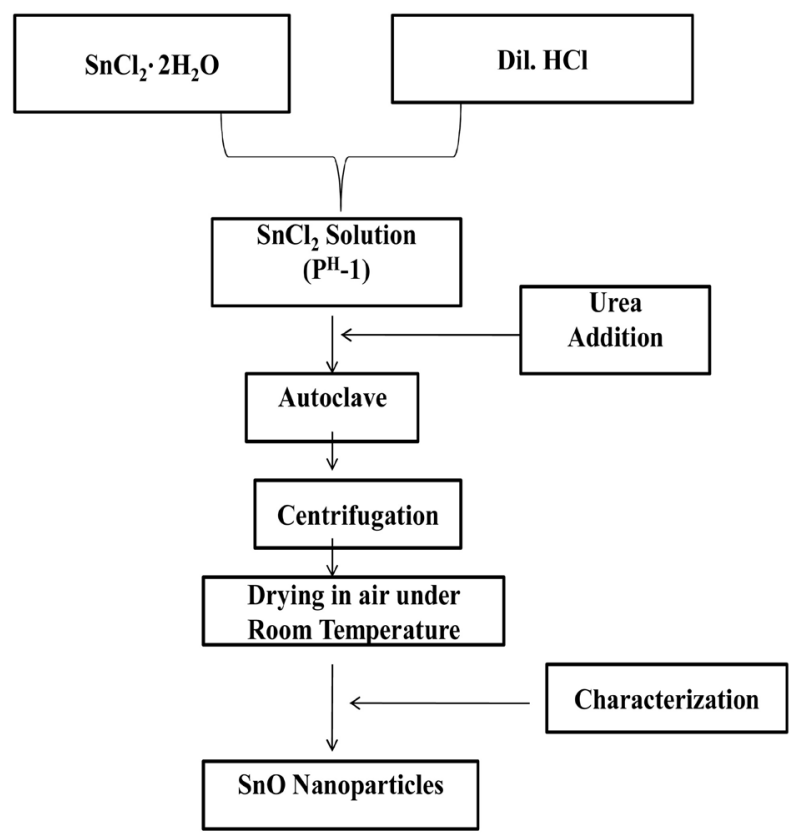

Figure 1. Flowchart for the synthesis of SnO Nanoparticles. 


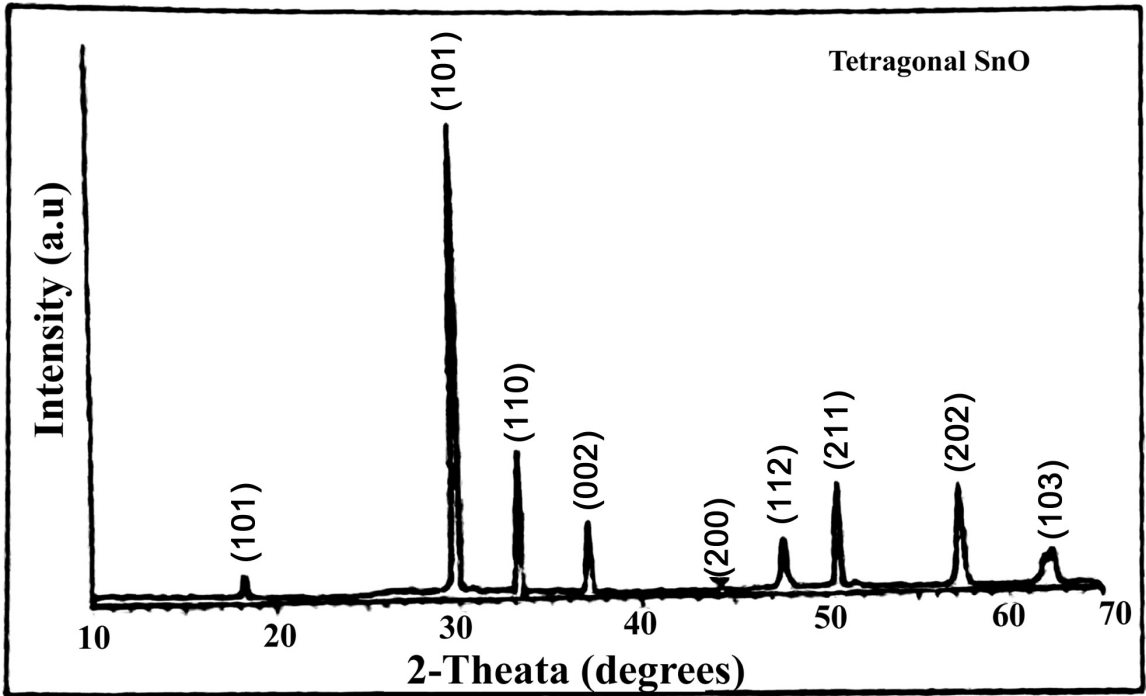

Figure 2. XRD of SnO Nanoparticles.

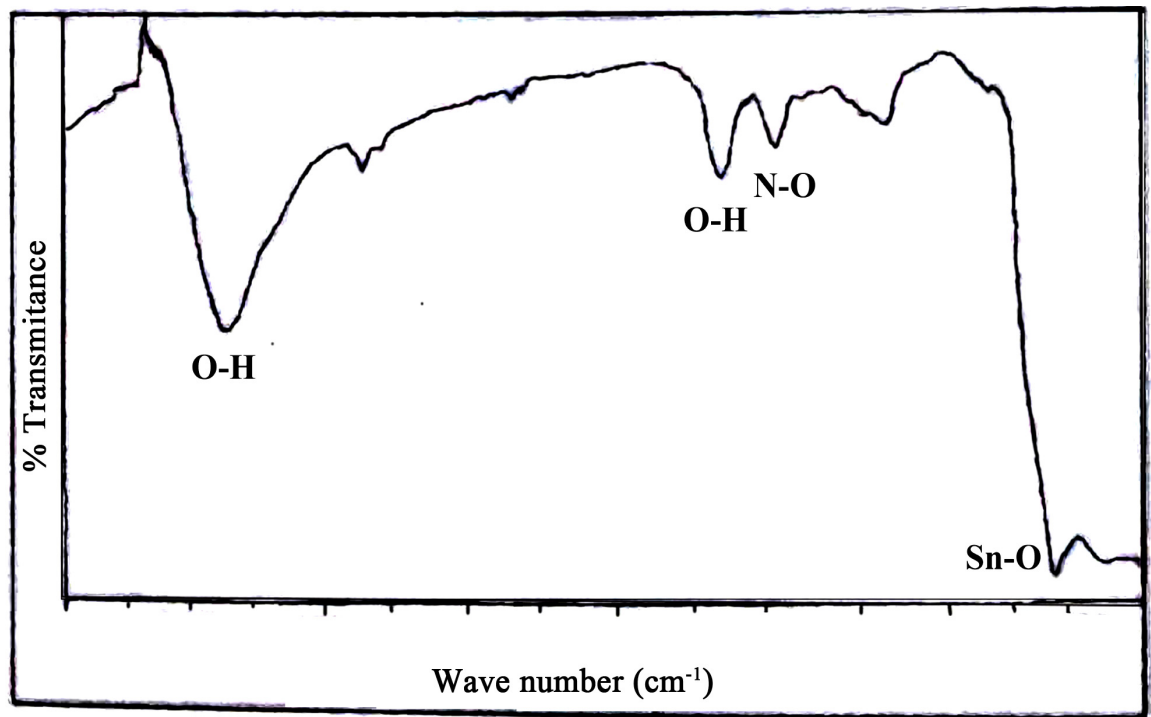

Figure 3. FT-IR spectrum of $\mathrm{SnO}$ nanoparticles.

$3456 \mathrm{~cm}^{-1}$ and $1618 \mathrm{~cm}^{-1}$ are attributed mainly to the $\mathrm{O}-\mathrm{H}$ stretching vibration of surface hydroxyl group or adsorbed water on the SnO nanoparticles. Peak observed at $1409 \mathrm{~cm}^{-1}$ is assigned to N-O. This may be from urea used in the experiment. The absorption band at $515 \mathrm{~cm}^{-1}$ is assigned to $\mathrm{Sn}-\mathrm{O}$ vibration.

\subsection{SEM Micrograph of SnO Nanoparticles}

The SEM micrograph of SnO nanoparticles is shown in Figure 4. It is seen that the particles are mesoporous in nature with particle size of $\sim 50 \mathrm{~nm}$.

\section{Conclusion}

In the present communication, nanosized particles of $\mathrm{SnO}$ were successfully synthesized by hydrothermal process using tin chloride. 


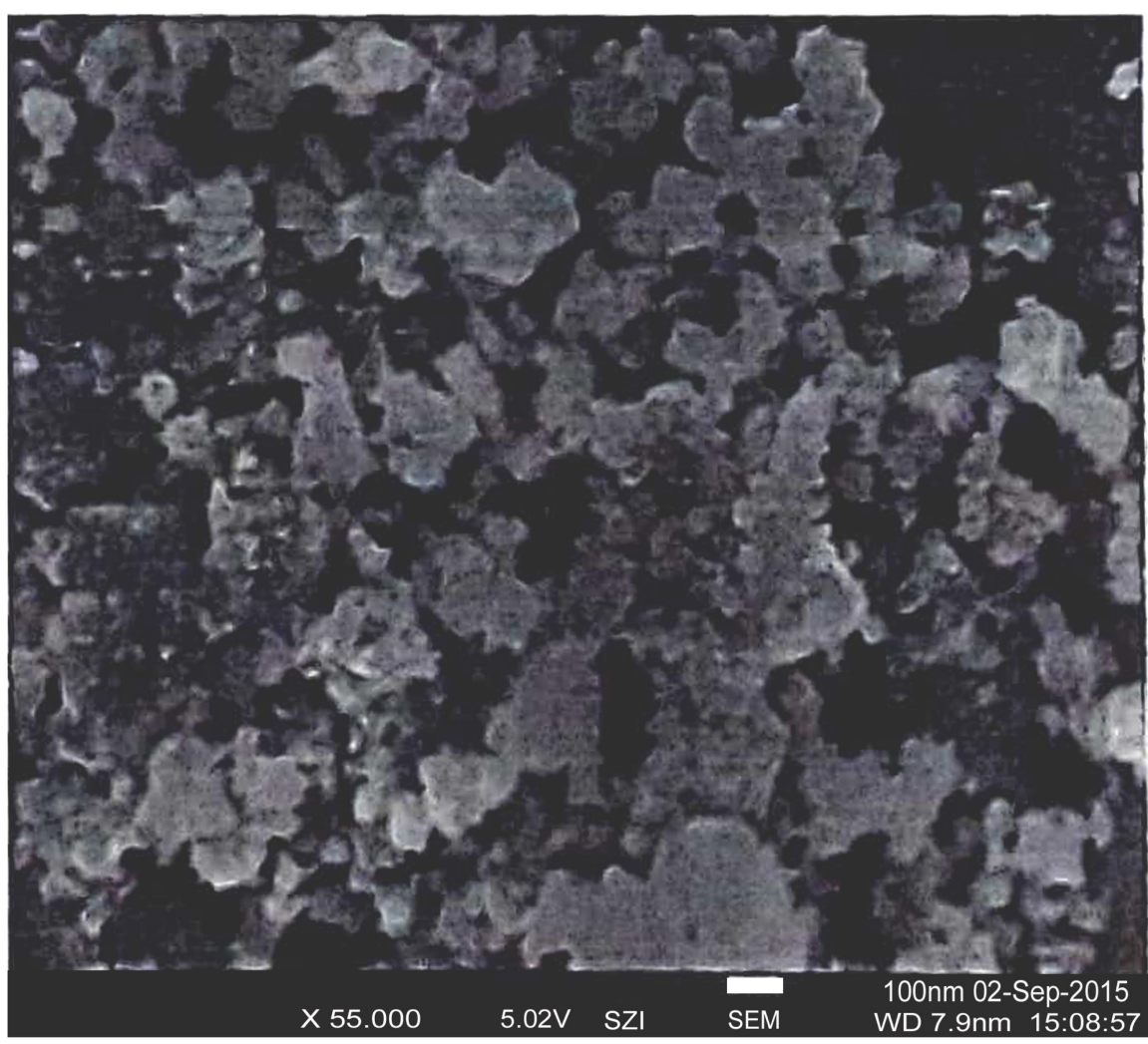

Figure 4. SEM micrograph of SnO nanoparticles.

\section{Acknowledgements}

Authors are thankful to Management, Executive Director, Principal and Head, Department of Science and Humanities for their encouragement and grants to carry out this research work.

\section{References}

[1] Jung, J. and Perrut, M. (2001) Particle Design Using Supercritical Fluids: Literature and Patent Survey. Journal of Supercritical Fluids, 20, 179-219. https://doi.org/10.1016/S0896-8446(01)00064-X

[2] Zhang, Y. and Erkey, C. (2006) Preparation of Supported Metallic Nanoparticles Using Supercritical Fluids: A Review. Journal of Supercritical Fluids, 38, 252-267. https://doi.org/10.1016/j.supflu.2006.03.021

[3] Reverchon, E., Adami, R. and Caputo, G. (2006) Supercritical Assisted Atomization: Performance Comparison between Laboratory and Pilot Scale. Journal of Supercritical Fluids, 37, 298-306.

[4] Adschiri, T., Hakuta, Y., Sue, K. and Arai, K. (2001) Hydrothermal Synthesis of Metal Oxide Nanoparticles at Supercritical Conditions. Journal of Nanoparticle Research, 3, 227-235. https://doi.org/10.1023/A:1017541705569

[5] Adschiri, T., Hakuta, Y. and Arai, K. (2000) Hydrothermal Synthesis of Metal Oxide Fine Particles at Supercritical Conditions. Industrial \& Engineering Chemistry Research, 39, 4901-4907. https://doi.org/10.1021/ie0003279

[6] Wang, W.-S., Zhen, L., Xu, C.-Y. and Shao, W.-Z. (2009) Room Temperature Synthesis, Growth Mechanism, Photocatalytic and Photoluminescence Properties of 
Cadmium Molybdate Core-Shell Microspheres. Crystal Growth \& Design, 9, 1558-1568.

[7] Mahlambi, M.M., Ngila, C.J. and Mamba, B.B. (2015) Recent Developments in Environmental Photocatalytic Degradation of Organic Pollutants: The Case of Titanium Dioxide Nanoparticles-A Review. Journal of Nanomaterials, 2015, Article ID: 790173.

[8] Zhang, S., Yin, Y., Gao, Y., Liu, Y., Qiu, F. and Wu, X. (2014) Modifying the Hierarchical Porosity of SBA-15 via Mild-Detemplation Followed by Secondary Treatments. Journal of Physical Chemistry, 2014, Article ID: 532317.

[9] Wang, J.X. Lu, C., Liu, X.C., Wang, Y.Q., Zhu, Z.X. and Meng, D.W. (2007) Nanostructures with Novel Distribution Characteristic and Superior Photocatalytic Performance. Materials \& Design, 115, 103-111.

[10] Wang, L., Shen, L., Zhu, L., Jin, H. and Bing, N. (2012) Preparation and Photocatalytic Properties of $\mathrm{SnO}_{2}$ Coated on Nitrogen-Doped Carbon Nanotubes. Journal of Nanomaterials, 2012, 6.

[11] Jiménez, V.M., González-Elipe, A.R., Espinós, J.P., Justo, A. and Ernández, A. (1996) Synthesis of $\mathrm{SnO}$ and $\mathrm{SnO}_{2}$ Nanocrystalline Powders by the Gas Phase Condensation Method. Sensors and Actuators B: Chemical, 31, 29-33.

[12] Cao, S.-W. and Zhu, Y.-J. (2008) Hierarchically Nanostructured $\alpha$ - $\mathrm{Fe}_{2} \mathrm{O}_{3}$ Hollow Spheres: Preparation, Growth Mechanism, Photocatalytic Property and Application in Water Treatment. Journal of Physical Chemistry C, 112, 6253-6257. 